\title{
Florística de lianas em um fragmento de floresta estacional semidecidual, Parque Estadual de Vassununga, Santa Rita do Passa Quatro, SP, Brasil
}

\author{
Yara Junqueira de Azevedo Tibiriçá1 ${ }^{1}$ Luis Francisco Mello Coelho ${ }^{2}$ e Leila Cunha de Moura ${ }^{1,3}$
}

Recebido em 5/11/2004. Aceito em 13/09/2005

\begin{abstract}
RESUMO - (Florística de lianas em um fragmento de floresta estacional semidecidual, Parque Estadual de Vassununga, Santa Rita do Passa Quatro, SP, Brasil). Embora o conhecimento sobre a florística dos fragmentos de florestas estacionais semideciduais tenha crescido nos últimos anos, ainda sabe-se pouco sobre a comunidade de lianas (lenhosas ou herbáceas) nesses fragmentos. Assim, foi realizado o levantamento florístico de lianas na gleba Maravilha, pertencente ao Parque Estadual de Vassununga (Santa Rita do Passa Quatro, SP), a fim de colaborar com o conhecimento dessa comunidade e subsidiar futuros trabalhos que envolvam essa forma de vida. A área de estudo compreende 127,08 ha, com inverno seco e temperatura média anual de $22{ }^{\circ} \mathrm{C}$. Para a coleta do material, percorreu-se mensalmente toda a borda do fragmento e três trilhas no interior da mata, de agosto/2002 a setembro/2003. Foram identificadas 120 espécies de lianas, pertencentes a 30 famílias e 71 gêneros, das quais $51 \%$ das espécies são volúveis, $42 \%$ apresentam gavinhas e apenas $7 \%$ são escandentes. As famílias mais representativas em número de espécies foram: Bignoniaceae (26), Malpighiaceae (14), Sapindaceae (12) e Asteraceae (9). Houve baixa similaridade florística entre as espécies de lianas presentes na gleba Maravilha em relação a outras áreas de florestas estacionais semideciduais do interior paulista.
\end{abstract}

Palavras-chave: lianas, trepadeiras, floresta estacional semidecidual, Parque Estadual de Vassununga, florística

\begin{abstract}
Floristic of lianas in a fragment of seasonal semidecidual forest State Park of Vassununga, Santa Rita do Passa Quatro, São Paulo State, Brazil). Although the knowledge about the floristic composition of the fragments of seasonal semidecidual forest had grown in the last few years, little is known about the liana communities (woody vines and herbaceous vines) in those fragments. To collaborate with the knowledgement of the lianas and subsidize future works involving this life form, a floristic survey of the liana species occurring at the fragment Maravilha of the State Park of Vassununga (Santa Rita do Passa Quatro - SP) was carried out. The study area comprised 127.08ha, with average temperature of $22^{\circ} \mathrm{C}$. The whole border of the forest fragment and three tracks inside the forest were surveyed monthly between August 2002 and September 2003. One hundred and twenty species of lianas, belonging to 30 families and 71 genera were identified where $51 \%$ of them were twiners, $42 \%$ tendrilous and $7 \%$ scandents. The most representative families in number of species were: Bignoniaceae (26), Malpighiaceae (14), Sapindaceae (12) and Asteraceae (9). Fragment Maravilha given low similarity with other fragments of semidecidual forest in São Paulo State.
\end{abstract}

Key words: lianas, seasonal semidecidual forest, floristic, fragmentation, tropical forest

\section{Introdução}

O interesse dos pesquisadores pelas espécies de plantas escaladoras tem crescido a partir das últimas décadas, sendo raros os estudos anteriores a 1980. Em sua maioria, estudos abordando composição e distribuição de espécies de lianas foram desenvolvidos em áreas de florestas pouco perturbadas ou contínuas (Putz 1984; Putz \& Chai 1987; Nabe-Nielsen 2001; Peres-Salicrup et al. 2001), conhecendo-se muito pouco sobre comunidades de lianas em fragmentos florestais (Avalos \& Mulkey 1999; Tabanez \& Viana 2000).
Segundo a classificação de Veloso (1992), lianas são todas as plantas lenhosas ou herbáceas que vivem apoiadas em outras plantas ou substrato, cujas gemas, acima do solo, são protegidas por catáfilos. Lianas diferenciam-se quanto ao modo de escalada (Gentry 1991), podendo ser classificadas em volúveis, com gavinhas (preensoras) e não preensoras (Hegarty 1991; Udulutsch et al. 2004).

Contudo, há de considerar-se que esta classificação muitas vezes torna-se complicada, pois diversas espécies combinam mais de um método de escalada, como é o caso de algumas espécies de Bignoniaceae e Leguminosae. Já outras espécies das famílias

\footnotetext{
1 Universidade Estadual Paulista, Instituto de Biociências, Departamento de Ecologia, Av. 24-A, CEP 13506-900, Rio Claro, SP, Brasil

2 Universidade Estadual Paulista, Instituto de Biociências, Departamento de Botânica, Av. 24-A, CEP 13506-900, Rio Claro, SP, Brasil

3 Autor para correspondência: lcmoura@unesp.rc.br
} 
Cucurbitaceae e Passifloraceae utilizam apenas um mecanismo de ascensão (Hegarty 1991).

Lianas são componentes florestais que contribuem significativamente para a diversidade de espécies tropicais (Gentry \& Dodson 1987). Há pelo menos 133 famílias que incluem espécies de lianas, no entanto, a maioria das espécies concentra-se em poucas famílias. Enquanto algumas famílias possuem apenas um ou dois gêneros de lianas, outras, como Bignoniaceae, Apocynaceae, Leguminosae, Malpighiaceae e Sapindaceae, são compostas predominantemente por gêneros de lianas (Gentry 1991).

Os levantamentos florísticos de lianas em florestas estacionais semideciduais do Estado de São Paulo (Hora \& Soares 2002; Udulutsch et al. 2004), encontraram o padrão descrito por Gentry (1991), no qual a maior parte das espécies de lianas concentra-se em poucas famílias. Contudo, ainda são poucos os trabalhos que abordam espécies de lianas (Hora \& Soares 2002).

Gentry (1991) ressaltou que a falta de conhecimento da florística de lianas é agravada, pois os levantamentos florísticos que abrangem todas as formas de vida dificilmente amostram boa parte das lianas, já que estas plantas são mais difíceis de serem vistas por ocuparem o dossel florestal (Nadkarni et al. 2001), merecendo uma amostragem direcionada e intensa.

Alguns autores (Putz 1984; Leitão Filho \& Morellato 1995; Tabanez \& Viana 2000) têm enfatizado a importância de se estudar a comunidade de lianas, visando um melhor manejo e conservação dos fragmentos florestais, pois diversos trabalhos destacam a invasão de lianas como um problema para a manutenção do fragmento, interferindo na regeneração da comunidade arbórea e aumentando a probabilidade de queda de árvores (Putz 1984). Por outro lado, os padrões fenológicos das lianas são, geralmente, complementares aos das árvores, resultando em constante suplemento de néctar, pólen e fruto para a fauna em períodos de escassez de frutos de espécies arbóreas (Morellato \& Leitão Filho 1996). Dessa forma, o levantamento florístico das espécies de lianas, assim como de outras formas de vida do fragmento, é o primeiro passo para a realização de um manejo bem sucedido sem que haja prejuízo para a fauna e flora e, conseqüentemente, perda de biodiversidade.

Levando-se em consideração os aspectos acima abordados, este estudo teve os objetivos de realizar o levantamento das espécies de lianas na gleba Maravilha do Parque Estadual de Vassununga e avaliar a similari- dade florística de lianas desse fragmento com outras áreas de florestas estacionais semideciduais, visando contribuir para o manejo do Parque e outros estudos.

\section{Material e métodos}

Área de Estudo - O presente trabalho foi desenvolvido na gleba Maravilha, situada no Parque Estadual de Vassununga $\left(21^{\circ} 41^{\prime}-44^{\prime}\right.$ S e 47³4' - 39'W), município de Santa Rita do Passa Quatro, nordeste do Estado de São Paulo. O Parque foi criado em 1970 e é composto por seis glebas isoladas, que somadas totalizam 1.732,14 ha (Secretaria do Meio Ambiente de São Paulo 1998). A cobertura vegetal predominante, com exceção da gleba Pé de Gigante (cerrado), é classificada como floresta estacional semidecidual (Veloso 1992).

O clima da região pelo sistema de classificação de Köppen (1948) é do tipo Cwag', ou seja, temperado, macrotérmico, moderadamente chuvoso com o inverno seco não rigoroso, com temperatura anual média de $22{ }^{\circ} \mathrm{C}$.

Nessa região, segundo Massoli (1981), predominam as Cuestas Basálticas, constituídas de arenitos das formações Botucatu, Pirambóia e Santa Rita, e pelos basaltos da formação Serra Geral. O solo da gleba Maravilha é composto por, pelo menos, três unidades: Latossolo Vermelho Escuro (predominantemente), Latossolo Roxo e, em menor proporção, Litossolo (Domingues et al. 1987).

A gleba Maravilha cobre uma área de 127,08 ha sendo delimitada pelo rio Mogi-Guaçu (40\% da borda), por monoculturas de cana-de-açúcar (cerca de 50\%) e pelo parque industrial da Usina Santa Rita de Açúcar e Álcool (10\%), estando sob forte pressão de degradação.

$\mathrm{O}$ remanescente florestal estudado encontra-se em uma área de encosta, com um desnível de 80 metros, apresentando diferenças na fisionomia, com algumas áreas degradadas e outras em melhor estado de conservação. Em geral, o estrato herbáceoarbustivo é ralo e lianas são muito freqüentes, dificultando a movimentação no interior e provocando queda de árvores.

Vieira et al. (1990), avaliando as principais espécies arbóreas da gleba Maravilha, amostraram 63 espécies. As espécies Metrodorea nigra A. St.-Hil. e Esenbeckia febrifuga (A. St.-Hil.) A. Juss. ex Mart. tiveram os maiores valores de densidade, Cariniana legalis (Mart.) O. Kuntze o maior valor de dominância, e Astronium graveolens Jacq., Metrodorea nigra, Aspidosperma ramiflorum Müll. Arg. e Holocalx 
balansae Mich. destacaram-se como as mais freqüentes.

Procedimentos - Este levantamento florístico foi realizado de agosto/2002 a setembro/2003, efetuando-se coleta mensal de indivíduos de lianas em floração ou frutificação, tanto nas bordas do fragmento quanto no seu interior. Para tal, foi utilizado um barco de alumínio com motor de pôpa para percorrer a borda que faz divisa com o rio Moji-Guaçu, sendo que o restante da área de borda e três trilhas no interior da mata foram percorridos em caminhadas. Todos os percursos foram realizados ao menos uma vez por mês, com exceção da mata ciliar, que foi percorrida apenas seis vezes durante o estudo, pois nos meses mais secos a navegação era inviável.

Considerando-se as diversas propostas de classificação dos modos de ascensão das lianas e a falta de um consenso por parte dos autores, optou-se pela classificação utilizada por Udulutsch et al. (2004) adaptada de Kelly (1985) e Hegart (1991), considerando volúveis as lianas que se enrolam em torno de um suporte por meio de ramos, caules e, mais raramente, pecíolos; lianas com gavinhas, plantas que se fixavam a um suporte por meio de estruturas modificadas em gavinhas de origem diversa (caulinar, foliar, etc.); e, lianas não preensoras ou passivas, as plantas que não possuíam estruturas específicas de fixação ou sensibilidade em órgãos, tais como ramos ou pecíolos, que se enrolam sobre a planta suporte (forófito), e escalavam apoiando-se passivamente sobre um suporte, podendo ser confundidas com plantas eretas em campo.

Para evitar problemas na classificação quanto ao modo de escalada, foram feitas observações em campo, anotando-se o principal modo de ascensão das espécies. Ainda assim, as dúvidas de classificação foram levadas a especialistas.

Os exemplares foram identificados por meio de chaves e descrições, comparação com materiais no Herbário do Instituto de Biociências da Unesp de Rio Claro (HRCB) e consulta a especialistas. Todo material herborizado foi incorporado ao HRCB e ao Laboratório de Ecologia do Instituto de Biociências (Unesp - Rio Claro). O sistema de classificação adotado foi o de Cronquist (1981).

Para análise da similaridade florística com outros fragmentos de florestas estacionais semideciduais do interior paulista foi utilizado o índice de Jaccard (Krebs 1998), considerando-se apenas as lianas determinadas até o nível de espécie.

\section{Resultados e discussão}

Foram identificadas 120 espécies de lianas, pertencentes a 71 gêneros e 30 famílias (Tab. 1). Dentre estas, Ipomoea sp. tem a possibilidade de ser uma espécie não descrita (Rosângela Simão-Bianchini, comunicação pessoal).

O número de espécies de lianas encontrado neste estudo foi próximo ao observado em outros fragmentos florestais no interior paulista. Entretanto, Udulutsch et al. (2004) detectaram um número maior de espécies (148) na mata São José, que pode estar relacionado ao maior esforço amostral realizado por estes pesquisadores, ou ainda, ao histórico de degradação da área, fertilidade do solo e heterogeneidade do fragmento.

R.G. Udulutsch (dados não publicados) realizou levantamento na Estação Ecológica de Caetetus, que contém um grande remanescente de floresta estacional semidecidual (2.178,84 ha), encontrando apenas 76 espécies. Tal fato poderia sugerir que em fragmentos maiores, e possivelmente melhor conservados, o número de espécies de lianas tende a ser menor. Porém, esta relação pode não ser verdadeira, pois os métodos aplicados e os esforços amostrais neste estudo e nos demais foram diferentes. Além disso, os levantamentos florísticos com ênfase em lianas são raros e não existem informações suficientes sobre a riqueza de espécies de lianas em áreas bem conservadas de florestas estacionais semideciduais para comparação com áreas degradadas, não sendo possível afirmar que há uma relação entre número de espécies e o estado de conservação da floresta.

As famílias que apresentaram maior riqueza de espécies na gleba Maravilha foram Bignoniaceae (26), seguida por Malpighiaceae (14), Sapindaceae (12), Asteraceae (nove), Convolvulaceae (sete), Apocynaceae (sete), Fabaceae (cinco), Cucurbitaceae (cinco), Menispermaceae e Dioscoreaceae, ambas com três espécies.

As 10 famílias mais freqüentes na gleba Maravilha, entre as que apresentam espécies de lianas, totalizaram $75,8 \%$ das espécies encontradas e correspondem a apenas 33,3\% das famílias estudadas, demonstrando que apesar do elevado número de espécies de lianas estas concentram-se em poucas famílias, reafirmando os estudos de Gentry (1991), Hora \& Soares (2002), R.G. Udulutsch (dados não publicados) e Udulutsch et al. (2004). Gentry (1991) relatou que a concentração das espécies em poucas famílias deve-se ao fato de ter ocorrido radiação evolutiva das espécies em poucos taxa. Segundo o mesmo autor, apesar de 133 famílias 
Tabela 1. Espécies e famílias de lianas amostradas em fragmento de floresta estacional semidecidual, Gleba Maravilha (Parque Estadual de Vassununga) Santa Rita do Passa Quatro, SP, Brasil e respectivo modo de ascensão. Gav. = com gavinhas; NP = não preensora; Vol. = volúvel.

Famílias/Espécies

Modo de

ascensão

\section{ALSTROEMERIACEAE}

Bomaria sp.

AMARANTHACEAE

Chaminossoa macrocarpa Kunth

Hebanthe paniculata Mart.

\section{APOCYNACEAE}

Condylocarpon isthmicum (Vell.) A. DC.

Forsteronia pilosa (Vell.) Müll. Arg.

F. pubescens A. DC.

F. refracta Müll. Arg.

Forsteronia sp.

Peltastes sp.

Prestonia coalita (Vell.) Woodson

\section{ASCLEPIADACEAE}

Calotropis procera $\mathrm{R}$. Br.

Funastrum clausum (Jacq.) Schlt.

ASTERACEAE

Baccharis flexuosa Baker

Dasyphyllum sp.

Mikania glomerata Spreng.

M. lundiana DC.

M. micrantha Kunth

M. ramosissima Gardner

Mikania sp.

Senecio cf. benthamii Sch. Bip. ex Wedd.

Vernonia scorpiodes (Lam.) Pers.

BIGNONIACEAE

Adenocalymma bracteatum (Cham.) A. DC.

A. marginatum (Cham.) A. DC.

A. paulistarum Bureau \& K. Schum.

A. paniculatum (L.) Kunth

A. chamberlaynii (Sims) Bureau \& K. Schum.

Arrabidaea chica (Bonpl.) Verl.

A. florida DC.

A. pulchella (Cham.) Bureau

A. selloi (Spreng.) Sandwith

Arrabidaea sp.

Clytostoma binatum (Thumb.) Sandw.

C. campanulatum (Cham.) Bureau \& K. Schum.

C. sciuripabulum Bureau \& K. Schum.

Cuspidaria floribunda (A. DC.) A.H. Gentry

Distictella elongata (Vahl) Urb.

Lundia obliqua Sond.

Macfadyena molli (Sond.) Seem.

M. unguis-cati (L.) A.H. Gentry

Mansoa difficilis (Cham.) Bureau \& K. Schum

Paragonia pyramidata (Rich.) Bureau

Pithecoctenium crucigerum (L.) A.H. Gentry

Pleonotoma cf. tetraquetrum (Cham.) Bureau

Pyrostegia venusta (Ker-Gawl.) Miers

Stizophyllum perforatum (Cham.) Miers

Tynnanthus fasciculatus (Vell.) Miers

T. micranthus Corr. Mello ex K. Shum
Vol.

NP

Vol.

Vol.

Vol.

Vol.

Vol.

Vol.

Vol.

Vol.

Vol.

Vol.

NP

Vol.

Vol.

Vol.

Vol.

Vol.

Vol.

Vol.

NP

Gav.

Gav.

Gav.

Gav.

Gav.

Gav.

Gav.

Gav.

Gav.

Gav.

Gav.

Gav.

Gav.

Gav.

Gav.

Gav.

Gav.

Gav.

Gav.

Gav.

Gav.

Gav.

Gav.

Gav.

Gav.

Gav.
Famílias/Espécies

Modo de

ascensão

\section{BORAGINACEAE \\ Cordia sp.}

CACTACEAE

Pereskia aculeata Mill.

CAESALPINACEAE

Bauhinia siqueira Ducke Gav.

CONVOLVULACEAE

Ipomoea $\mathrm{sp}$.

I. alba $\mathrm{L}$.

I. hederifolia $\mathrm{L}$.

I. pes-caprae (L.) Sweet.

I. quamoclit $\mathrm{L}$.

I.tubata Ness.

Merremia macrocalyx (Ruiz \& Pav.) O' Donell

CUCURBITACEAE

Cayaponia sp.

Luffa ciilindrica $\mathrm{L}$.

Melothria cucumis Vell.

Momordica charantia L.

Psiguria ternata (Roem) C. Jeffrey

DILLENIACEAE

Davilla rugosa Poir.

Tetracera willdenowiana Steud.

DIOSCOREACEAE

Dioscorea monadelpha (Kunth) Grisseb.

D. multiflora Mart. \& Grisseb.

Dioscorea sp.

EUPHORBIACEAE

Dalechampia pentaphylla Lam. Vol.

D. stipulaceae Müll. Arg.

Vol.

NP

Vol.

Vol.

Vol.

Vol.

Vol.

Vol.

Vol.

Gav.

Gav.

Gav.

Gav.

Gav.

Vol.

Vol.

Vol.

Vol.

Vol.

Vol.
Vol.

\section{FABACEAE}

Centrosema sp.

Machaerium oblogifolium Vogel

Machaerium sp.

Vol.

NP

Gav.

Phaseolus sp.1

Phaseolus sp. 2

HIPPOCRATEACEAE

Hemiangium excelsum A.C. Smith

Hippocratea decussata (Ruiz \& Pav.) O’Donell

MALPIGHIACEAE

Banisteriopsis adenopoda (A. Juss.) B. Gates

B. lutea (Griseb.) Cuatrec.

B. oxyclada (A. Juss.) B. Gates

Dicella bracteosa (A. Juss.) Griseb.

Heteropterys pauciflora A. Juss.

Mascagnia andiphia (A. Juss.) Griseb.

M. anisopetala (A. Juss.) Griseb.

M. chlorocarpa (A. Juss.) Griseb.

M. a cordifolia (A. Juss.) Griseb.

Stigmaphyllon tomentosum A. Juss.

S. lalandianum A. Juss.

Tetrapterys guilleminiana A. Juss.

T.s multiglandulosa A. Juss.

T. ramiflora A. Juss
Vol.

Vol.

Vol

Vol.

Vol.

Vol.

Vol.

Vol.

Vol.

Vol.

Vol.

Vol.

Vol.

Vol.

Vol.

Vol.

Vol.

Vol.

continua 
Tabela 1 (continuação)

\begin{tabular}{lc}
\hline Famílias/Espécies & $\begin{array}{c}\text { Modo de } \\
\text { ascensão }\end{array}$ \\
\hline MENDONCIACEA & \\
$\quad$ Mendocia sp. & Vol. \\
MENISPERMACEAE & \\
$\quad$ Cissampelos pareira L. & Vol. \\
Odontocarya sp.1 & Vol. \\
Odontocarya sp.2 & Vol. \\
MIMOSACEAE & \\
Acacia paniculada Willd. & Vol. \\
PASSIFLORACEAE & \\
$\quad$ Passiflora sp. & Gav. \\
RANUNCULACEAE & \\
$\quad$ Clematis dioica L. & Vol. \\
RHAMNACEAE & \\
$\quad$ Gouania virgata Reissek & Gav. \\
RUBIACEAE & \\
Manettia luteo-rubra (Vell.) Benth. & Vol. \\
Manettia sp. & Vol. \\
SAPINDACEAE & \\
Paullinia elegans Cambess. & Gav. \\
P. meliaefolia A. Juss. & Gav. \\
P. rhomboidea Radlk. & Gav. \\
\hline
\end{tabular}

terem representantes de plantas escaladoras, 64\% das espécies de lianas neotropicais encontram-se em apenas 12 famílias e 26 famílias incluem $85 \%$ do total de espécies de lianas do Novo Mundo.

As famílias Bignoniaceae, Sapindaceae e Malpighiaceae, apesar de nem sempre nessa ordem, também estiveram entre as três famílias mais ricas em outros levantamentos de lianas realizados no interior paulista (Morellato \& Leitão Filho 1996; Rezende \& Hora \& Soares 2002; R.G. Udulutsch, dados não publicados e Udulutsch et al. 2004; Ranga 2005). Segundo Gentry (1991), as famílias Bignoniaceae, Sapindaceae e Malpighiaceae são compostas principalmente por gêneros de lianas, sendo muito expressivas nas regiões de baixas altitudes do Novo Mundo, fator que explica a alta prevalência dessas nos levantamentos florísticos referentes à comunidade de lianas, realizados em florestas estacionais semideciduais.

A família Bignoniaceae foi a mais representativa quanto ao número de espécies. Esta família apresenta maior riqueza na maioria dos levantamentos florísticos de plantas escaladoras na região neotropical (Gentry 1991). Mesmo assim, a taxonomia da família Bignoniaceae no Brasil é pouco estudada, abordando preferencialmente as espécies arbóreas, sendo as lianas

\begin{tabular}{|c|c|}
\hline Famílias/Espécies & $\begin{array}{l}\text { Modo de } \\
\text { ascensão }\end{array}$ \\
\hline Serjania caracasana (Jacq.) Willd. & Gav. \\
\hline S. fuscifolia Radlk. & Gav. \\
\hline S. laruotteana Cambess. & Gav. \\
\hline S. lethalis A. St.-Hil. & Gav. \\
\hline S. meridionalis Cambess. & Gav. \\
\hline S. paradoxa Radlk. & Gav. \\
\hline Urvillea laevis Radlk. & Gav. \\
\hline U. ulmacea Kunth & Gav. \\
\hline \multicolumn{2}{|l|}{ SMILACACEAE } \\
\hline Smilax sp. 1 & Gav. \\
\hline Smilax sp. 2 & Gav. \\
\hline \multicolumn{2}{|l|}{ SOLANACEAE } \\
\hline Solanum sp. 1 & NP \\
\hline Solanum sp. 2 & NP \\
\hline \multicolumn{2}{|l|}{ STERCULIACEAE } \\
\hline Byttneria catalpifolia Jacq. & NP \\
\hline \multicolumn{2}{|l|}{ ULMACEAE } \\
\hline Celtis iguanae (Jacq.) Sarg. & NP \\
\hline \multicolumn{2}{|l|}{ VITACEAE } \\
\hline Cissus sp. 1 & Gav. \\
\hline Cissus sp. 2 & Gav. \\
\hline
\end{tabular}

raramente tratadas (Scudeller \& Carvalho-Okano 1998).

Gentry (1976) analisou a distribuição de espécies pertencentes à família Bignoniaceae em diversos ecossistemas na América Central, notando que em todos eles a média de espécies encontradas foi em torno de 20 espécies, incluindo arbóreas e lianas. O número de espécies desta família encontrado em nosso estudo e nos demais no interior paulista aqui analisados, com exceção de Rezende \& Ranga (2005) que encontrou 13 espécies, foi igual ou maior, mesmo restringindo-se às espécies arbóreas e arbustivas (Morellato \& Leitão Filho 1996; Hora \& Soares 2002; R.G. Udulutsch, dados não publicados; Udulustch et al. 2004), alcançando 29 espécies em um mesmo fragmento. Dessa forma, este trabalho corrobora os demais estudos em fragmentos de florestas estacionais semideciduais, apontando a família Bignoniaceae como a mais expressiva em número de espécies.

A segunda família mais representativa na gleba Maravilha foi Malpighiaceae, que é composta principalmente por lianas e apresenta ampla distribuição nas regiões tropicais (Joly 2002). Neste levantamento foram coletadas 14 espécies, mostrando a grande representatividade dessa família na gleba Maravilha, condizendo com outros trabalhos em fragmentos de 
florestas estacionais semideciduais no interior do Estado de São Paulo (Morellato \& Leitão Filho 1996; Hora \& Soares 2002; R.G. Udulustsch, dados não publicados; Udulustsch et al. 2004), os quais também obtiveram um número elevado de espécies de lianas da família Malpighiaceae.

A família Sapindaceae, constituída principalmente por lianas, compreende cerca de 140 gêneros (Cronquist 1981; Joly 2002). Das 12 espécies de Sapindaceae encontradas na gleba Maravilha, metade é do gênero Serjania.

O gênero Serjania possui aproximadamente 226 espécies, sendo que das 92 ocorrentes no Brasil (47 endêmicas), 36 estão no planalto central, das quais 11 são endêmicas da região e três ocorrem apenas em São Paulo, tendo como habitat principal margens de rios e áreas degradadas (Acevedo-Rodríguez 1990). Das espécies encontradas na gleba Maravilha S. paradoxa é endêmica do Brasil, e apesar de Acevedo-Rodríguez (1990) ter restringido sua área de ocorrência ao habitat de campo, nosso levantamento acrescenta ambientes florestais à sua área de ocorrência.

Além das três famílias citadas anteriormente, Gentry (1991) destacou a família Asclepiadaceae como sendo uma das mais bem representadas entre as lianas do Novo Mundo (Gentry 1991), estando também entre as seis famílias mais ricas de lianas encontradas na Mata Atlântica do Estado de São Paulo (A.C. Kim, dados não publicados). Porém, tanto este levantamento, no qual só foram amostradas duas espécies de lianas pertencentes à família Asclepiadaceae, quanto nos demais, realizados em florestas estacionais semideciduais, que identificaram, em média, 4 espécies (Morellato \& Leitão Filho 1996; Hora \& Soares 2002; Udulutsch et al. 2004; Rezende \& Ranga 2005), sugerem que nos fragmentos desse bioma a família Asclepiadaceae apresenta menor destaque do que registrado por Gentry (1991) e A.C. Kim (dados não publicados) nos neotrópicos e na Mata Atlântica.

Dentre as lianas estudadas na Gleba Maravilha, pouco mais da metade das espécies $(51 \%)$ foram classificadas como volúveis, $42 \%$ apresentaram gavinhas e apenas $7 \%$ foram consideradas como escandentes.

A maior parte das famílias (52\%) possui espécies exclusivamente volúveis (que alcançam as copas das árvores enrolando-se em algum suporte), 24\% das famílias incluem espécies de lianas que são unicamente escandentes, $14 \%$ apresentam espécies com gavinhas como único método de escalada, enquanto $7 \%$ das famílias possuem tanto espécies de lianas volúveis quanto espécies de lianas com gavinhas e apenas $3 \%$ das famílias possuem espécies de lianas com os três métodos de escalada.

O predomínio de espécies e famílias com lianas volúveis e com gavinhas, e um menor número de espécies escandentes, foi sugerido por Udulutsch et al. (2004) como sendo um provável padrão para florestas estacionais semideciduais do Estado de São Paulo. Os mesmos autores ressaltaram a necessidade de novos estudos para comprová-lo. Os resultados do presente estudo aumentam a probabilidade da existência do padrão sugerido.

A comunidade de lianas da Gleba Maravilha apresentou baixa similaridade florística com outros remanescentes de florestas estacionais semideciduais. A Estação Ecológica do Noroeste Paulista, São José do Rio Preto / Mirassol (Rezende \& Ranga 2005) foi a mais diferenciada com $14,8 \%$ de similaridade; em segundo lugar ficou a Fazenda Canchim em São Carlos (Hora \& Soares 2002) com apenas 16,2\%. Santa Genebra, Campinas (Morellato \& Leitão Filho 1996) também foi muito pouco semelhante com a Gleba Maravilha (18,1\%), seguida pelo levantamento realizado na Estação Ecológica dos Caetetus, municípios de Gália e Alvilândia (R.G. Udulutsch, dados não publicados)

Tabela 2. Riqueza de espécies de lianas em fragmentos de floresta estacional semidecidual do Estado de São Paulo e respectivos números de espécies em comum e índices de similaridade (Jaccard) em relação à Gleba Maravilha, Parque Estadual de Vassununga, Santa Rita do Passa Quatro, SP, Brasil.

\begin{tabular}{lccccc}
\hline Local de estudo & $\begin{array}{c}\text { Área } \\
\text { (ha) }\end{array}$ & $\begin{array}{c}\text { Número de } \\
\text { espécies }\end{array}$ & $\begin{array}{c}\text { Número de } \\
\text { espécies em comum }\end{array}$ & $\begin{array}{c}\text { Similaridade } \\
\text { (J) }\end{array}$ & Referência \\
\hline Estação Ecológica dos Caetetus & $2.178,84$ & 76 & 37 & 0,23 & R.G. Udulutsch, dados não publicados \\
Santa Genebra & 250 & 136 & 39 & 0,18 & Morellato \& Leitão Filho 1996 \\
Fazenda Cachim & 112 & 109 & 32 & 0,16 & Hora \& Soares 2002 \\
Mata São José & 230 & 148 & 62 & 0,30 & Udulutsch et al. 2004 \\
Estação Ecológica Noroeste & 168,6 & 105 & 29 & 0,15 & Rezende \& Ranga 2005 \\
Gleba Maravilha & 127,08 & 120 & - & - & Presente estudo \\
\hline
\end{tabular}


com 23\% de similaridade e pela Mata São José (Udulutsch et al. 2004), que foi o fragmento com mais espécies de lianas em comum com a Gleba Maravilha, com $30 \%$ de similaridade (Tab. 2).

A similaridade florística entre as espécies de lianas da gleba Maravilha com os demais fragmentos de florestas estacionais semideciduais do interior paulista (Morellato \& Leitão Filho 1996; Hora \& Soares 2002; Udulustsch et al. 2004; Rezende \& Ranga 2005) foi, no geral, baixa. A baixa similaridade na composição florística entre fragmentos ocorre, entre outras causas, porque várias espécies têm hábitat preferencial distintos, assim a fragmentação pode ocasionar extinções diferenciais em função das variações ambientais, mesmo em fragmentos relativamente próximos (Bernacci \& Leitão Filho 1996).

À medida que aumenta a fragmentação, cresce também a perda de diversidade e algumas espécies passam a ocorrer em apenas alguns poucos fragmentos, aumentando a importância preservacionista e conservacionista dessas áreas (Bernacci \& Leitão Filho 1996). Mesmo reduzida, a diversidade de espécies nos fragmentos florestais ainda é considerável e a destruição desses remanescentes florestais causaria uma inevitável perda da diversidade genética e biológica (Bernacci \& Leitão Filho 1996). Estudos como estes são fundamentais e devem ser incentivados, pela sua importância para o entendimento da dinâmica de remanescentes florestais.

\section{Agradecimentos}

À Fundação "O Boticário de Proteção à Natureza", pelo financiamento da pesquisa; à "Idea Wild", pela doação de material; aos especialistas Dr. Marco Antonio de Assis (Bignoniaceae), Dr. João Semir (Asteraceae), Dra. Maria Cândida H. Mamede (Malpighiaceae), Dra. Rosângela Simão-Bianchini (Convolvulaceae), Renata Udulutsch e Dra. Maria Silva Ferrucci (Sapindaceae), pelo auxílio na identificação.

\section{Referências bibliográficas}

Acevedo-Rodríguez, P. 1990. Distributional patterns in Brazilian Serjania (Sapindaceae). Acta Botanica Brasilica 4(1): 69-82.

Avalos, G. \& Mulkey, S.S. 1999. Seasonal changes in liana cover in the upper canopy of a neotropical dry forest. Biotropica 31(1): 186-192.
Bernacci, L.C. \& Leitão Filho, H.F. 1996. Flora fanerogâmica da floresta da Fazenda São Vicente, Campinas, SP. Revista Brasileira de Botânica 19(2): 149-164.

Cronquist, A. 1981. An integrated system of classification of flowering plants. New York, Columbia University Press.

Domingues, E.N.; Silva, D.A. \& Pfeifer, R.M. 1987. Reconhecimento da geomorfologia e solos do Parque Estadual de Vassununga, SP. Boletim Técnico 41(2): 271-290.

Gentry, A.H. 1976. Bignoniaceae of Southern Central America: Distribution and Ecological Specificity. Biotropica 8(2): 117-131.

Gentry, A.H. 1991. The distribution and evolution of climbing plants. Pp. 3-53. In: F.E. Putz \& H.A. Mooney. The Biology of Vines. Cambridge, Cambridge University Press.

Gentry, A.H. \& Dodson, C. 1987. Contribuition of nontrees to species richness of a tropical rain forest. Biotropica 19(2): 149-156.

Hegarty, E.E. 1991. Vine-host interactions. Pp. 3-53. In: F.E. Putz \& H.A. Mooney. The Biology of Vines. Cambridge, University Press.

Hora, R.C. \& Soares, J.J. 2002. Estrutura fitossociológica da comunidade de lianas em uma floresta estacional semidecidual na Fazenda Canchim, São Carlos, SP. Revista Brasileira de Botânica 25(3): 323-329.

Joly, A.B. 2002. Botânica: Introdução à taxonomia vegetal. $13^{a}$ ed. São Paulo, Companhia Editora Nacional.

Kelly, D.L. 1985. Epiphytes and climbers of a Jamaican rain forest: vertical distribution, life forms and life histories. Journal of Biogeography 12: 223-241.

Köeppen, W. 1948. Climatologia. México, Buenos Aires, Ed. Fondo de Cultura Econômica.

Krebs, C.J. 1998. Ecological Methodology. $2^{\text {nd }}$ ed. University of British Columbia, Ed. Bejamin Cummings.

Leitão Filho, H. \& Morellato, P. 1995. A vegetação da Reserva de Santa Genebra. P. Morellato \& H.F. Leitão Filho (orgs.). In: Ecologia e preservação de uma floresta tropical urbana, Reserva de Santa Genebra. Campinas, Editora da UNICAMP.

Massoli, M. 1981. Geologia do Município de Santa Rita do Passa Quatro - SP. Revista do Instituto Geológico 2(2): $35-45$.

Morellato, P.C. \& Leitão Filho, H.F. 1996. Reroductive phenology of climbers in a Southeastem Brazilian forest. Biotropica 28(2): 180-191.

Nabe-Nielsen, J. 2001. Growth and mortality rates of the liana Machaerium cuspidatum in relation to light and topographic position. Biotropica 34(2): 319-322.

Nadkarni, N.M.; Merwin, M.C. \& Nieder, J. 2001. Forest canopies plant diversity. In: Encyclopedia of biodiversity. New York, Academic Press 3.

Peres-Salicrup, D.; Sork, V.L. \& Putz, F.E. 2001. Lianas and trees in a liana forest of Amazonian Bolivia. Biotropica 33(1): 34-47.

Putz, F.E. 1984. The natural history of lianas on Barro Colorado Island, Panama. Ecological Society of America 65(6): 1713-1724. 
Putz; F.E. \& Chai, P. 1987. Ecological studies of lianas in Lambir National Park, Sarawak, Malaysia. Journal of Ecology 75: 523-531.

Rezende, A.A. \& Ranga, N.T. 2005. Lianas da Estação Ecológica do Noroeste Paulista, São José do Rio Preto/ Mirassol, SP, Brasil. Acta Botanica Brasilica 19(2): 273-279.

Scudeller, V.V. \& Carvalho-Okano, R.M. 1998. Bignonieae (Bignoniaceae) no Parque Estadual do Rio Doce, Minas Gerais, Brasil. Iheringia, Sér. Bot., 51(1): 79-133.

Secretaria do Meio Ambiente de São Paulo. 1998. Atlas das Unidades de Conservação Ambiental do Estado de São Paulo. Parte II: Interior-São Paulo. Metalivros (ed.). São Paulo, Câmara Brasileira do Livro

Tabanez, A.A. \& Viana, V.M. 2000. Patch structure whithin brazilian Atlantic Forest fragments and implications for conservation. Biotropica 32(4b): 925-933.
Udulutsch, R.G.; Assis, M.A. \& Picchi, D. 2004. Florística de trepadeiras numa floresta estacional semidecídua, Rio Claro - Araras, Estado de São Paulo, Brasil. Revista Brasileira de Botânica 27(1): 125-134

Veloso, H.P. 1992. Sistema fitogeográfico. Pp. 9-38 In Fundação Instituto Brasileiro de Geografia e Estatística. Manual Técnico da Vegetação Brasileira. Rio de Janeiro (Manuais Técnicos em Geociências, 1).

Vieira, M.G.L.; Bertoni, J.E.A.; Moraes, J.L. \& Zandarin, M. 1990. A. Análise das principais espécies arbóreas ocorrentes na Gleba Maravilha do Parque Estadual de Vassununga - SP, n. VI. Pp. 626-629. In: Congresso Florestal Brasileiro. Campos do Jordão, 1990. São Paulo, IF Ed. 